\title{
Diagnostic accuracy of preoperative ultrasonography in predicting contralateral inguinal hernia in children: a systematic review and meta-analysis
}

\author{
K. M. A. Dreuning ${ }^{1}$ • C. E. M. ten Broeke ${ }^{1}$ • J. W. R. Twisk ${ }^{2}$ - S. G. F. Robben ${ }^{3}$ - R. R. van Rijn ${ }^{4}$ - J. I. M. L. Verbeke $^{5}$ \\ L. W. E. van Heurn ${ }^{1}$. J. P. M. Derikx ${ }^{1}$
}

Received: 21 April 2018 / Revised: 4 June 2018 / Accepted: 21 June 2018 / Published online: 27 July 2018

(C) The Author(s) 2018

\begin{abstract}
Objectives The incidence of children developing metachronous contralateral inguinal hernia (MCIH) is $7-15 \%$. Contralateral groin exploration during unilateral hernia repair can prevent MCIH development and subsequent second surgery and anaesthesia. Preoperative ultrasonography is a less invasive strategy and potentially able to detect contralateral patent processus vaginalis (CPPV) prior to $\mathrm{MCIH}$ development.

Methods We queried MEDLINE, Embase and Cochrane library to identify studies regarding children aged $<18$ years diagnosed with unilateral inguinal hernia without clinical signs of contralateral hernia, who underwent preoperative ultrasonography of the contralateral groin. We assessed heterogeneity and used a random-effects model to obtain pooled estimates of sensitivity, specificity and area under the receiver operating characteristic curve (AUC).

Results Fourteen studies (2120 patients) were included, seven (1013 patients) in the meta-analysis. In studies using surgical exploration as reference test $(n=4,494$ patients), pooled sensitivity and specificity were $93 \%$ and $88 \%$ respectively. In studies using contralateral exploration as reference test following positive and clinical follow-up after negative ultrasonographic test results $(n=3,519$ patients), pooled sensitivity was $86 \%$ and specificity $98 \%$. The AUC (0.984) shows high diagnostic accuracy of preoperative ultrasonography for detecting CPPV, although diagnostic ultrasonographic criteria largely differ and large heterogeneity exists. Reported inguinal canal diameters in children with CPPV were $2.70 \pm 1.17 \mathrm{~mm}, 6.8 \pm 1.3 \mathrm{~mm}$ and $9.0 \pm 1.9 \mathrm{~mm}$.

Conclusion Diagnostic accuracy of preoperative ultrasonography to detect CPPV seems promising, though may result in an overestimation of $\mathrm{MCIH}$ prevalence, since CPPV does not invariably lead to $\mathrm{MCIH}$. Unequivocal ultrasonographic criteria are mandatory for proper diagnosis of CPPV and subsequent prediction of MCIH.
\end{abstract}

Electronic supplementary material The online version of this article (https://doi.org/10.1007/s00330-018-5625-6) contains supplementary material, which is available to authorized users.

K. M. A. Dreuning

k.m.dreuning@amc.uva.nl

1 Department of Paediatric Surgery, Paediatric Surgical Center of Amsterdam, Emma Children's Hospital AMC \& VU University Medical Center, Meibergdreef 9, 1105

AZ Amsterdam, The Netherlands

2 Department of Methodology and Applied Biostatistics, and the Amsterdam Public Health Research Institute, VU University Medical Center, De Boelelaan 1089a, 1081 HV Amsterdam, The Netherlands
3 Department of Radiology, Maastricht University Medical Center, P. Debyelaan 25, 6229 HX Maastricht, The Netherlands

4 Department of Radiology, Academic Medical Center/Emma Children's Hospital, Meibergdreef 9, 1105

AZ Amsterdam, The Netherlands

5 Department of Radiology and Nuclear Medicine, VU University Medical Center, De Boelelaan 1117, 1081

HV Amsterdam, The Netherlands 


\section{Key Points}

- Diagnostic accuracy of preoperative ultrasonography for detection of CPPVin children with unilateral inguinal hernia is high.

- Preoperative ultrasonographic evaluation of the contralateral groin assumedly results in an overestimation of MCIH prevalence.

- Unequivocal ultrasonographic criteria are mandatory for proper diagnosis of CPPV and risk factor identification is needed to predict whether CPPV develops into clinically apparent MCIH.

Keywords Hernia, inguinal · Ultrasonography · Child

$\begin{array}{ll}\text { Abbreviations } & \\ \text { CPPV } & \text { Contralateral patent processus vaginalis } \\ \text { MCIH } & \text { Metachronous contralateral inguinal hernia } \\ \text { PRISMA } & \begin{array}{l}\text { Preferred Reported Items for Systematic } \\ \text { Reviews and Meta-Analysis statement }\end{array} \\ \text { PROSPERO } & \begin{array}{l}\text { International Prospective Register of } \\ \text { Systematic Reviews }\end{array} \\ \text { PV } & \begin{array}{l}\text { Processus vaginalis } \\ \text { Width of low echoic region of the internal ring }\end{array}\end{array}$

\section{Introduction}

Inguinal hernia is the most common surgical condition in childhood and presents in approximately $0.8-5 \%$ of term children and more than $30 \%$ in premature born children $[1,2]$. At the time of surgery, the hernia is unilateral in $80 \%$ of children. However, $7-15 \%$ of children develop a hernia on the opposite side after unilateral hernia repair, a metachronous contralateral inguinal hernia $(\mathrm{MCIH})$, with the highest risk in children under the age of 6 months or with initial left-sided hernia [3, 4]. Medical history and physical examination of the groin are not adequate to detect a hidden (or asymptomatic) patent processus vaginalis (PV) [5-7], which is likely to develop into an MCIH. MCIH necessitates a second operation and anaesthesia. The repeated use of anaesthetics possibly affect the development of children's brains [8]. Preventive strategies have been proposed since the 1950s and exploration of the contralateral groin during unilateral hernia repair is frequently used to detect contralateral patent processus vaginalis (CPPV). However, contralateral exploration carries risks of operative complications (e.g. wound infection, haematoma or testicular atrophy) and is unnecessary if the processus vaginalis is closed $[9,10]$. Therefore, management of the contralateral groin remains controversial.

If contralateral hernia development could be predicted prior to symptomatic hernia repair, individual recommendations regarding contralateral exploration can be made more consciously. Accurate preoperative evaluation of the contralateral inguinal canal could prevent unnecessary contralateral exploration and might reduce the incidence of children developing MCIH and obviating the need for a second operation and anaesthesia.
Previous studies show that preoperative ultrasonography has high accuracy for detecting both symptomatic and asymptomatic inguinal hernias in children, as the sensitivity for diagnosing CPPV is $98 \%$ and $97 \%$, respectively $[6,11,12]$. False negative rates of ultrasonography-based detection of CPPV are difficult to determine since most studies do not evaluate the contralateral inguinal canal intra- or postoperatively [13].

The aim of this systematic review and meta-analysis is to assess the diagnostic accuracy of preoperative ultrasonography of the contralateral groin to detect CPPV and its potential value to predict development of $\mathrm{MCIH}$ in children with unilateral inguinal hernia.

\section{Methods}

\section{Protocol}

A systematic review was conducted in accordance with the Preferred Reported Items for Systematic Reviews and MetaAnalysis (PRISMA) statement on all literature regarding preoperative ultrasonography as a diagnostic tool for contralateral inguinal hernia in children. The protocol was registered (PROSPERO 2017, CRD42017058269).

\section{Literature search}

We queried EMBASE, MEDLINE, PubMed, the Cochrane library databases and reference lists of eligible articles for the selection of studies. The search strategy was conducted in December 2016 (Appendix 1) and updated in November 2017.

\section{Eligibility criteria}

Articles were considered eligible irrespective of language or publication date and status. Inclusion criteria were (a) children under 18 years old diagnosed with unilateral inguinal hernia who had (b) no clinical signs of contralateral hernia and (c) underwent preoperative ultrasonography of the contralateral groin prior to surgical hernia repair. Patients diagnosed with 
(a) bilateral inguinal hernia or (b) inguinal hernia associated with non-descendent testis were excluded from data analysis.

\section{Study selection and methodological quality assessment}

Two reviewers independently performed the screening and selection of studies based on title and abstract, and full text for final selection. The full text of articles was retrieved by contacting the authors if it was not available via our library and attempts were made to translate articles that were not reported in English. The most complete study was included if the same data was repeatedly reported. Modified Quality Assessment of Diagnostic Accuracy Studies (QUADAS-2) tool was used to independently assess the methodological quality of the included studies. Inconsistencies were solved by second joint review of the literature or by consulting a third independent review author.

\section{Data extraction and analysis}

Appendix 2 comprises data that was systematically extracted from the studies included in this systematic review by the two reviewers and recorded in a data collection form. Missing data were calculated if possible and unpublished data or further details were retrieved by contacting study author(s).

Surgery, i.e. contralateral exploration, and clinical followup were used as the reference standard following positive index test results. Negative index test results were followed by surgery, clinical follow-up or no reference test. Studies that performed contralateral exploration in all patients, regardless of the index test results, are referred to as 'complete cases'. Studies that solely performed contralateral exploration following positive index test results are referred to as 'incomplete cases'.

\section{Statistical analysis}

Statistical analysis was performed using Review Manager (version 5.3.5) and Meta-DiSc (version 1.4). The available data were inserted in $2 \times 2$ tables to compute sensitivity, specificity, positive predictive value (PPV) and negative predictive value (NPV) for each study. A random-effects model was used to obtain pooled estimates of sensitivity and specificity including their $95 \%$ confidence intervals. Heterogeneity between studies was assessed using the $\chi^{2}$ and $I^{2}$ statistic. The summary receiver operating characteristic (SROC) curve was used to estimate the area under the curve (AUC) that represented overall diagnostic performance of the index test.

\section{Results}

\section{Literature search}

Literature search and manual reference analysis yielded 1346 potentially eligible studies. Duplicates were removed and 916 records were excluded after the initial screening. Nine more records were excluded after full text screening and 14 studies were included in this review (Fig. 1).

\section{Study characteristics}

The 14 studies were published between 1992 and 2017. They included 2120 children diagnosed with unilateral inguinal hernia undergoing preoperative ultrasonography of the contralateral groin. Study and index test characteristics are described in Tables 1 and 2. If preoperative ultrasonography yielded positive test results, contralateral exploration ( $n=12$ studies) and clinical follow-up ( $n=2$ studies) were performed, negative index test results were followed by contralateral exploration ( $n=4$ studies), clinical follow-up ( $n=7$ studies) or no other reference test ( $n=3$ studies). Generally, the index test (preoperative ultrasonography) was considered positive if there was flow of abdominal fluid or structures through an open PV or the diameter of the PV was more than $4 \mathrm{~mm}$; it was considered negative if the PV was closed or the diameter was less than 4 $\mathrm{mm}$. The flow chart in Appendix 3 shows how diagnostic test accuracy results were determined. Seven studies were included in the meta-analysis [14-20].

\section{Methodological quality}

Quality assessment using the QUADAS-2 tool indicated a moderate to high risk of bias concerning the selection of patients, because among others patient selection was not specified [18] and exclusions were made based on sex [5, 16, 21] or age $[15,17,22]$. The risk of bias regarding the flow and timing domain was also considered high as most studies limited the use of contralateral exploration as the reference standard to a preset part of their study population $[5,7,11,18-20,22,23]$. Assessment of bias regarding the index test domain and overall applicability concerns were considered low (Fig. 2a, b).

\section{Studies excluded from the meta-analysis}

Seven studies were excluded from the meta-analysis. In five studies, diagnostic accuracy test results could not be determined because no reference test was performed [5, 11, 22] or the diagnostic or reference test results could not be extracted, calculated or obtained from the authors $[7,23]$ (Table 3). Two studies were excluded because clinical follow-up was used as reference standard in all children, irrespective of the index test results. Their diagnostic accuracy test results were 
Fig. 1 PRISMA flow chart of the study selection

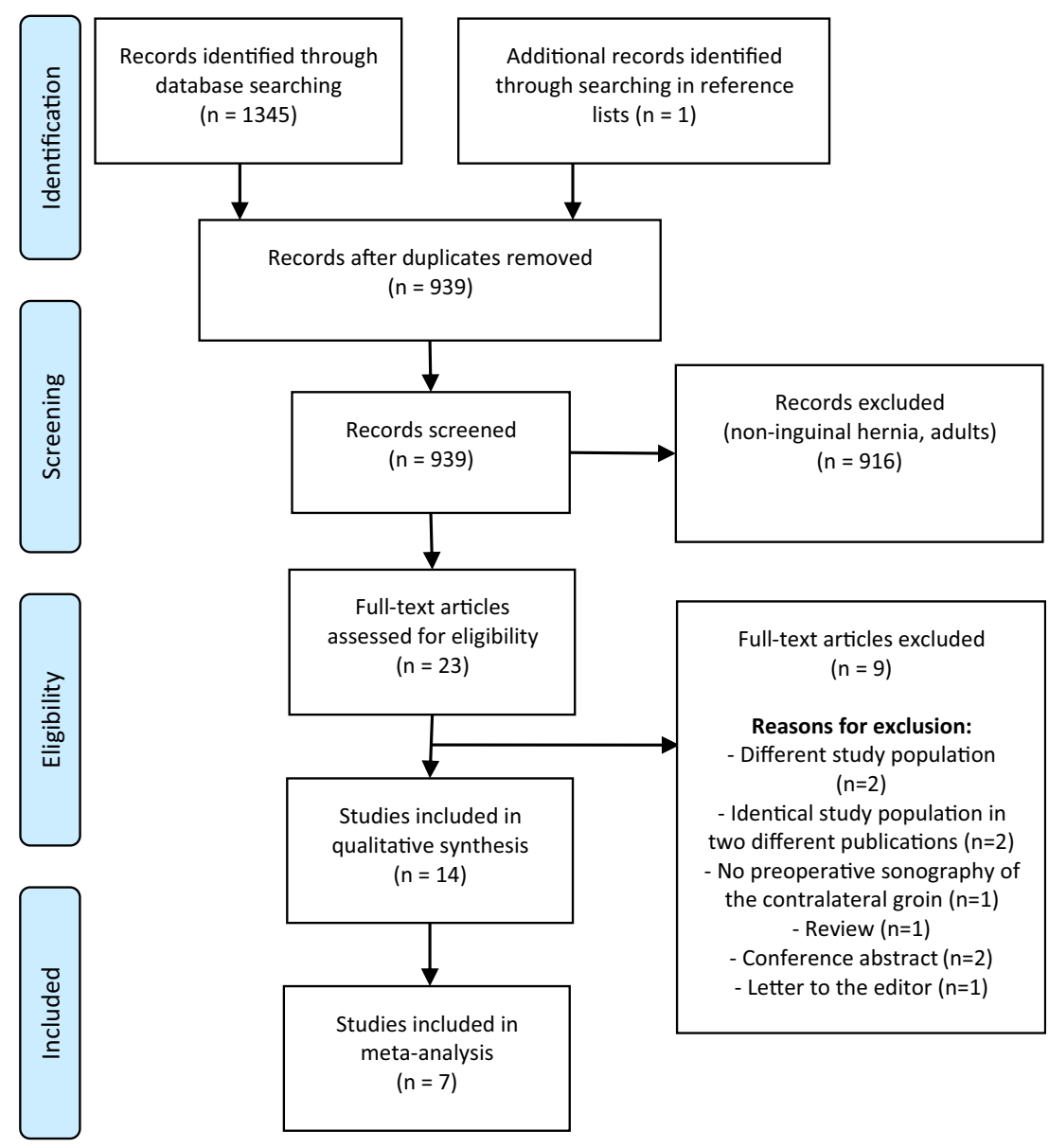

12 to 48 months. The diagnostic sensitivity ranged from $75 \%$ (at a specificity of $97 \%$ ) to $100 \%$ (at a specificity of almost 99\%) $[13,18-20]$. The pooled sensitivity was $86 \%$ (95\% CI $\left.78-92 \% ; \chi^{2}=17.07, p<0.01 ; I^{2}=88.3 \%\right)$ and the pooled specificity was $98 \%\left(95 \%\right.$ CI $96-99 \% ; \chi^{2}=1.15, p<0.01 ; I^{2}$ $=0.0 \%$ ) (Fig. 3b). The AUC of the SROC was 0.996 (Fig. 4b).

\section{Diameter of the width of low echoic region of the internal ring (WLIR) [19]}

Three studies including 424 children contributed data regarding the WLIR of the contralateral inguinal ring. In children without patency of the contralateral PV the mean diameter of the contralateral ring was $1.34 \pm 1.26 \mathrm{~mm}, 3.5 \pm 0.4 \mathrm{~mm}[13,20]$ or less than $6.0 \mathrm{~mm}$ in boys in particular [19]. The mean WLIR in children diagnosed as having a CPPV was $6.8 \pm 1.3 \mathrm{~mm}$ and in children diagnosed as having a contralateral hernia the mean WLIR was $2.70 \pm 1.17 \mathrm{~mm}$ or $9.0 \pm 1.9 \mathrm{~mm}[13,20]$.

\section{Discussion}

In this review, we aimed to assess the diagnostic accuracy of preoperative ultrasonography of the contralateral groin to the meta-analysis (Table 5). The follow-up period ranged from 


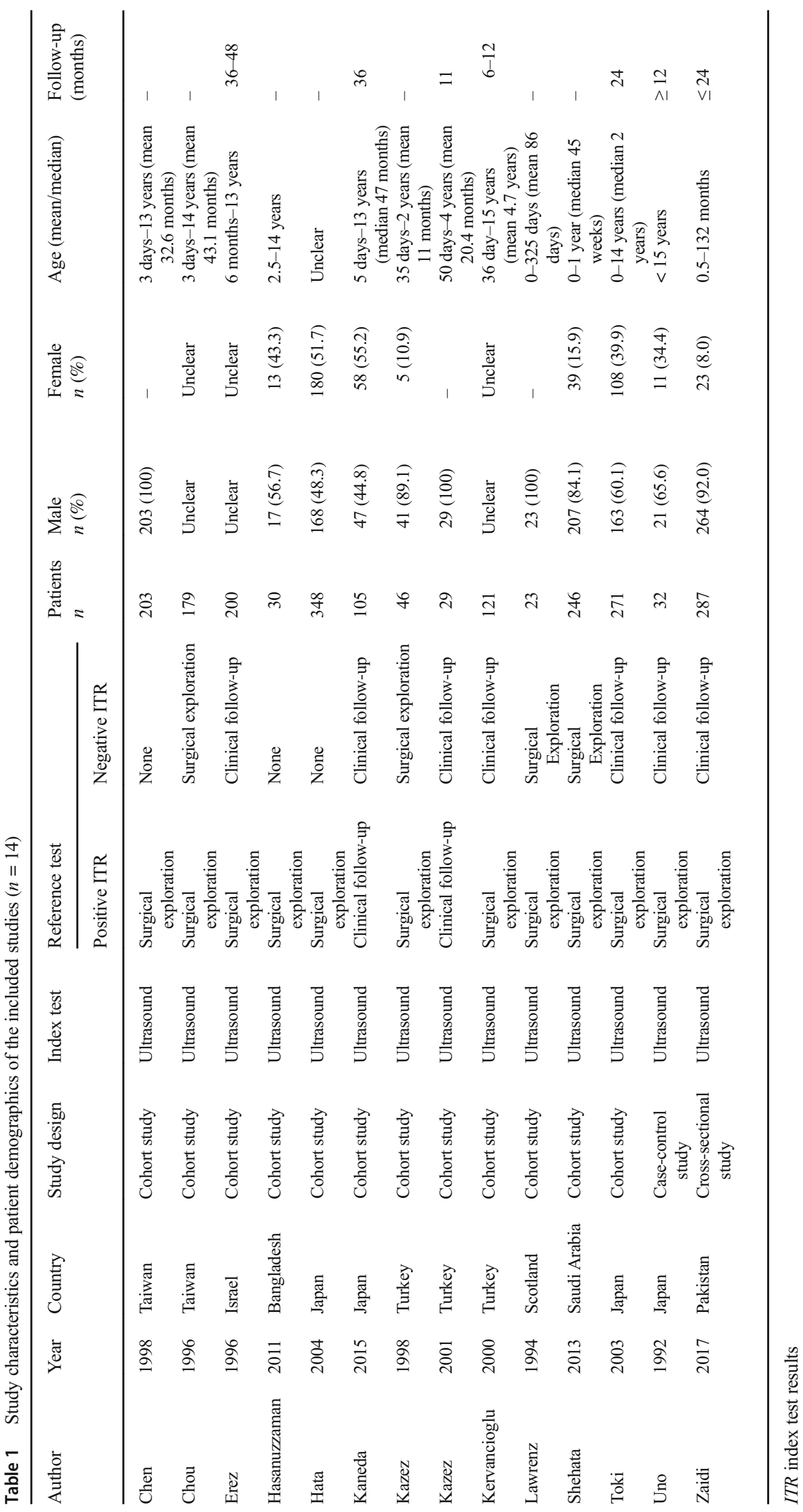




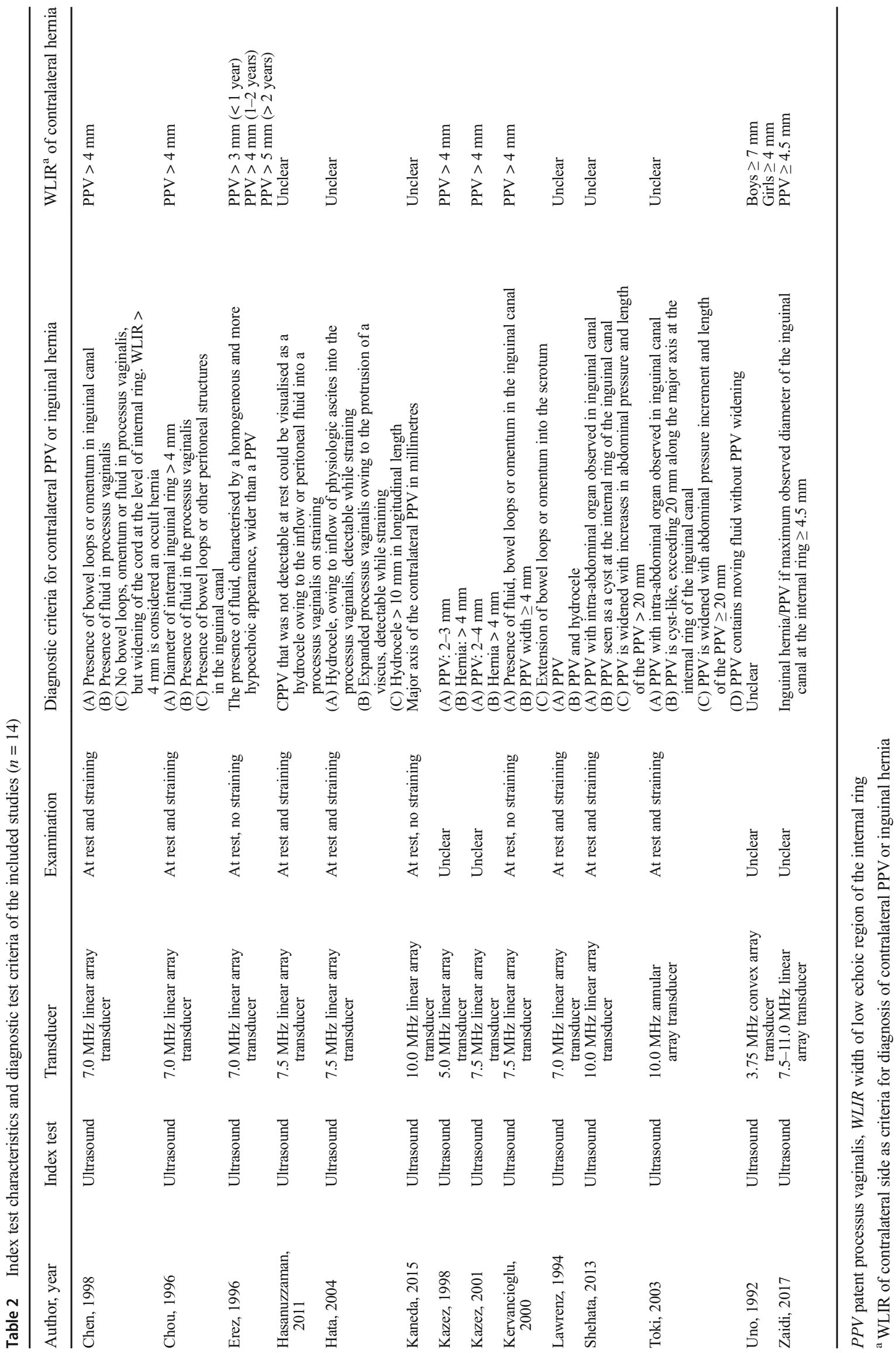


Fig. 2 Methodological quality assessment of the included studies using the QUADAS-2 tool. a Risk of bias and applicability concerns summary about each domain are shown for each included study; $\mathbf{b}$ risk of bias and applicability concerns about each domain presented as percentages across included studies. Red circle with minus sign, high risk; yellow circle with question mark, unclear risk; green circle with plus sign, low risk

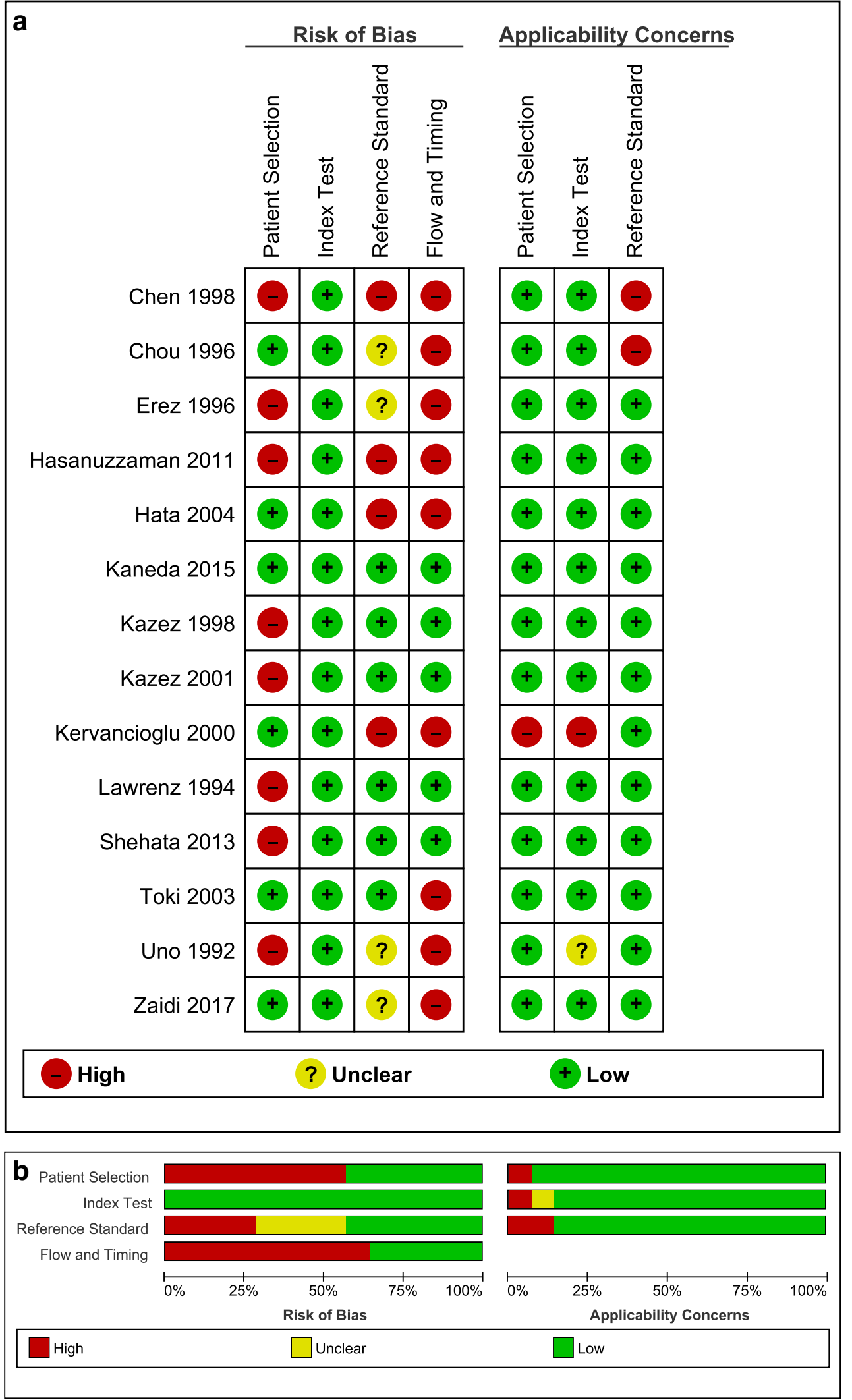

detect CPPV and its potential value to predict MCIH in children with unilateral inguinal hernia.
Seven studies were included in this meta-analysis. They were subdivided into two groups: 'complete' and 
Table 3 Diagnostic accuracy test results from studies that were excluded from the meta-analysis $(n=7)$

\begin{tabular}{|c|c|c|c|c|c|c|c|c|c|c|c|c|}
\hline Author, year & $\begin{array}{l}\text { Patients } \\
(n)\end{array}$ & TP & FP & $\mathrm{FN}$ & $\mathrm{TN}$ & $\begin{array}{l}\text { Follow-up } \\
\text { (months) }\end{array}$ & $\begin{array}{l}\text { Sensitivity } \\
(\%)\end{array}$ & $\begin{array}{l}\text { Specificity } \\
(\%)\end{array}$ & $\begin{array}{l}\text { PPV } \\
(\%)\end{array}$ & $\begin{array}{l}\text { NPV } \\
(\%)\end{array}$ & $\begin{array}{l}\text { US CPPV/MCIH } \\
(\%)\end{array}$ & $\begin{array}{l}\text { Clinical CPPV/ } \\
\text { MCIH (\%) }\end{array}$ \\
\hline Chen, 1998 & 203 & 62 & 3 & - & - & - & - & - & 95.4 & - & 32.0 & - \\
\hline $\begin{array}{l}\text { Hasanuzzaman, } \\
2011\end{array}$ & 30 & 11 & 1 & - & - & - & - & - & 91.7 & - & 40.0 & - \\
\hline Hata, 2004 & 348 & 74 & 4 & - & - & - & - & - & 94.9 & - & 22.4 & - \\
\hline Kaneda, 2015 a & 105 & 9 & 27 & 2 & 67 & 36 & 81.8 & 71.3 & 25 & 97.1 & 34.3 & 10.5 \\
\hline Kazez, $2001^{\mathrm{a}}$ & 29 & 0 & 11 & 0 & 18 & 11 & - & 62 & - & 100 & 37.9 & 0 \\
\hline $\begin{array}{l}\text { Kervancioglu, } \\
2000\end{array}$ & 121 & 10 & 0 & - & - & $6-12$ & - & - & 100 & - & 8.3 & - \\
\hline Toki, 2003 & 271 & - & - & 4 & - & 24 & - & - & - & - & - & - \\
\hline
\end{tabular}

$T P$ true positive, $F P$ false positive, $F N$ false negative, $T N$ true negative, $P P V$ positive predictive value, $N P V$ negative predictive value, $U S$ ultrasound, $C P P V$ contralateral patent processus vaginalis, $M C I H$ metachronous contralateral inguinal hernia

${ }^{\text {a }}$ Clinical findings during follow-up were used to calculate the amount of true positives (TP), false positives (FP), false negatives (FN) and true negatives $(\mathrm{TN})$

'incomplete' cases. Studies referred to as complete cases reported both the positive and negative index test results of contralateral exploration and showed high pooled sensitivity $(93 \%)$ and moderate pooled specificity $(88 \%)$. Incomplete cases reported only the positive index test results of contralateral exploration and the negative index test results of the findings of clinical followup, and show lower pooled sensitivity (86\%) though higher pooled specificity (98\%). AUC implies that diagnostic performance of preoperative ultrasonography is very good at detecting CPPV in complete $(0.984)$ and incomplete cases $(0.996)$. These results indicate that preoperative ultrasonography is less likely to result in a high number of false negatives in the complete cases. In the incomplete cases, the false positive rate (FPR) is low $(2.1 \%)$ compared to the FPR of complete cases $(11.5 \%)$. Negative preoperative ultrasonographic test results yield positive perioperative findings during contralateral exploration more often compared to the amount of clinically apparent hernias that actually develop postoperatively. These results suggest that patency of the contralateral PV is much more often detected during contralateral exploration than an MCIH actually develops during follow-up.

It is reported that at first presentation, $40 \%$ of the children diagnosed with a unilateral inguinal hernia also have a patent PV on the contralateral side [24-26]. However, the process of obliteration of a patent PV continues in the first few months of life, causing a successive decrease in the PV patency incidence and thus the incidence of positive surgical findings with advancing age. A patent PV during contralateral exploration will therefore not necessarily develop into a clinically relevant inguinal hernia: the estimated childhood risk of developing a clinically apparent inguinal hernia in case of the presence of a patent PV is only between $25 \%$ and $50 \%$ [24]; however, it considerably increases the risk for $\mathrm{MCIH}$ development, and risk factors to predict whether a CPPV actually develops into an MCIH or not need to be identified. This corresponds to the results of Kaneda et al., who related both the positive and negative ultrasonographic test results to clinical follow-up
Table 4 Diagnostic accuracy test results from complete cases included in the meta-analysis $(n=4)$. Complete cases: all patients underwent both the index test (preoperative ultrasonography) and reference test (surgical exploration), irrespective of the index test results. Clinical findings during follow-up (if reported) were used to calculate the amount of false negatives $(\mathrm{FN})$ and true negatives $(\mathrm{TN})$

\begin{tabular}{lccccccccccc}
\hline Author, year & $\begin{array}{l}\text { Patients } \\
(n)\end{array}$ & TP & FP & FN & TN & $\begin{array}{l}\text { Sensitivity } \\
(\%)\end{array}$ & $\begin{array}{l}\text { Specificity } \\
(\%)\end{array}$ & $\begin{array}{l}\text { PPV } \\
(\%)\end{array}$ & $\begin{array}{l}\text { NPV } \\
(\%)\end{array}$ & $\begin{array}{l}\text { US CPPV }^{\mathrm{a}} \\
(\%)\end{array}$ & $\begin{array}{l}\text { OR CPPV }^{\mathrm{b}} \\
(\%)\end{array}$ \\
\hline Chou, 1996 & 179 & 49 & 11 & 0 & 119 & 100 & 91.5 & 81.7 & 100 & 33.5 & 27.4 \\
Kazez, 1998 & 46 & 23 & 2 & 1 & 20 & 95.8 & 90.9 & 92 & 95.2 & 54.3 & 52.2 \\
Lawrenz, 1994 & 23 & 14 & 4 & 4 & 1 & 77.8 & 20.0 & 77.8 & 20.0 & 78.3 & 78.3 \\
Shehata, 2013 & 246 & 75 & 20 & 7 & 144 & 91.7 & 87.7 & 75.9 & 96.2 & 38.6 & 33.3 \\
\hline
\end{tabular}

$T P$ true positive, $F P$ false positive, $F N$ false negative, $T N$ true negative, $P P V$ positive predictive value, $N P V$ negative predictive value, $U S$ ultrasound, $C P P V$ contralateral patent processus vaginalis

${ }^{\text {a }}$ Amount (\%) of contralateral patent processus vaginalis as determined by ultrasonography

${ }^{\mathrm{b}}$ Amount (\%) of contralateral patent processus vaginalis detected intraoperatively 
a
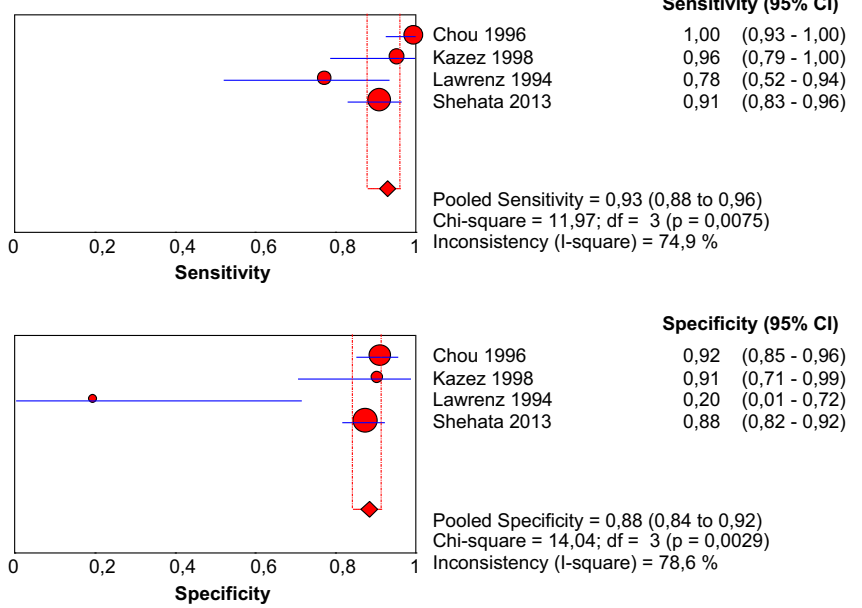

b
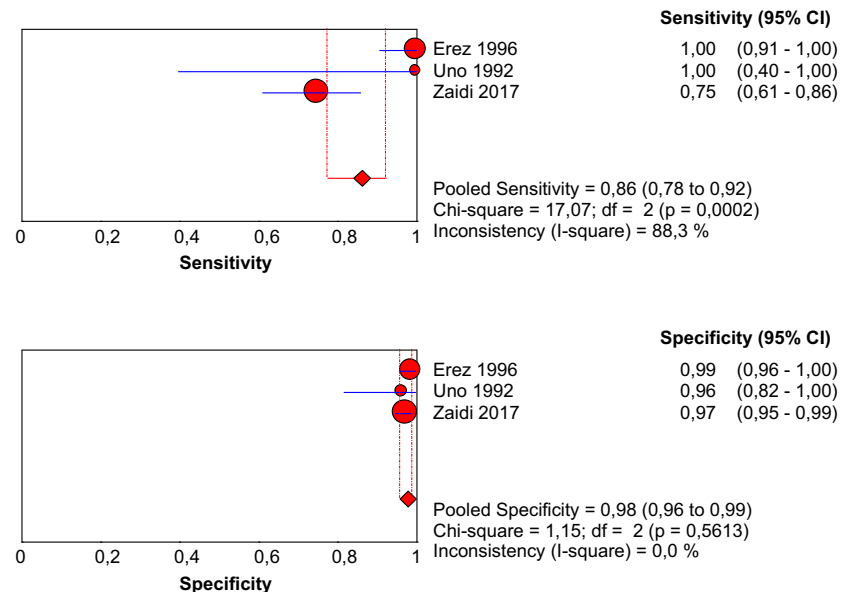

Fig. 3 Pooled sensitivity and specificity forest plots including the $95 \%$ confidence intervals (CI) of a complete cases and $\mathbf{b}$ incomplete cases

and reported a high 'false positive rate' of $75 \%$ (27/36). In contrast to the other studies included in this review, the number of true positives is extremely low (Tables 3, 4 and 5), implying that only $25 \%$ of the CPPVs actually developed into a clinically apparent MCIH. Prevalence of CPPV as determined by preoperative ultrasonography was $34.3 \%$, though after clinical follow-up the prevalence of $\mathrm{MCIH}$ decreased to $10.5 \%$ [13]. As the index test results are considered positive on the basis of the presence of CPPV, regardless whether it actually develops into a clinically apparent $\mathrm{MCIH}$ or not, one can assume that preoperative ultrasonography overestimates MCIH prevalence (Tables 2 and 3 ).

The preoperative major mean CPPV axis as determined using ultrasound was significantly wider in patients with contralateral inguinal hernia compared to patients without contralateral hernia. Unfortunately, there was great variability in the diameter of the contralateral WLIR among the included studies. The mean WLIR in children diagnosed with contralateral hernia or patent PV was $2.70 \pm 1.17 \mathrm{~mm}, 6.8 \pm 1.3 \mathrm{~mm}$ or $9.0 \pm 1.9$ $\mathrm{mm}[13,20]$, whereas in other studies children with a mean WLIR of $1.34 \pm 1.26 \mathrm{~mm}, 3.5 \pm 0.4 \mathrm{~mm}$ and less than $6.0 \mathrm{~mm}$ were diagnosed as having no patent PV $[13,19,20]$. Previously, Erez et al. prospectively compared the sonographic dimensions of the symptomatic, unilateral inguinal canal with the surgical findings in 642 children undergoing inguinal hernia repair and perioperatively found a PPV, 'full hernia' and 'large hernia' when a mean groin width of $4.9 \pm 1.1 \mathrm{~mm}, 7.2 \pm 2.0$ $\mathrm{mm}$ and $12.8 \pm 3.6 \mathrm{~mm}$ was ultrasonically detected [27].

One of the difficulties interpreting our study results is that the diagnostic ultrasonographic criteria to detect CPPV differ among the included studies (Table 2). First, some studies differentiate between CPPV and contralateral inguinal hernia,

\section{a}

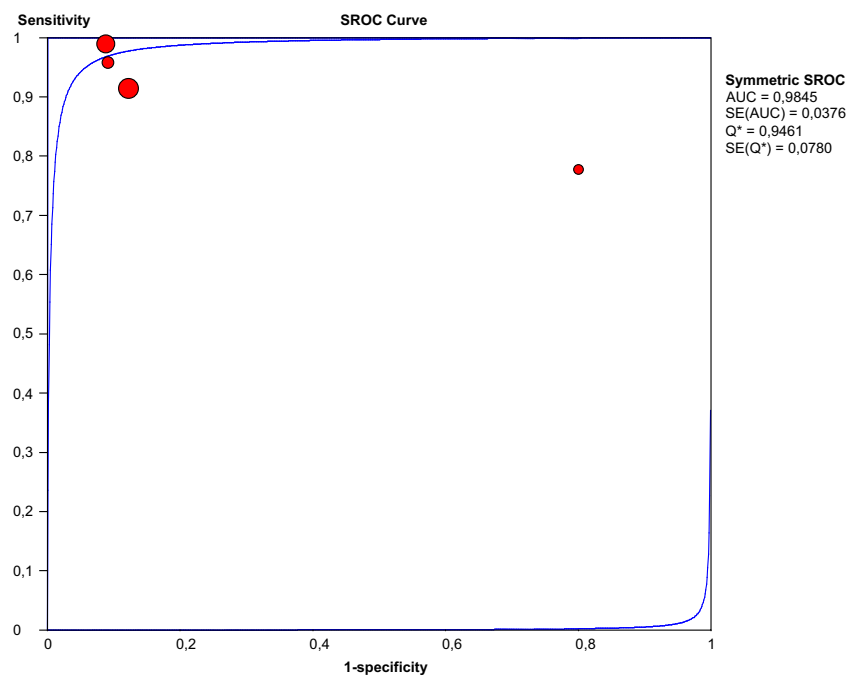

b

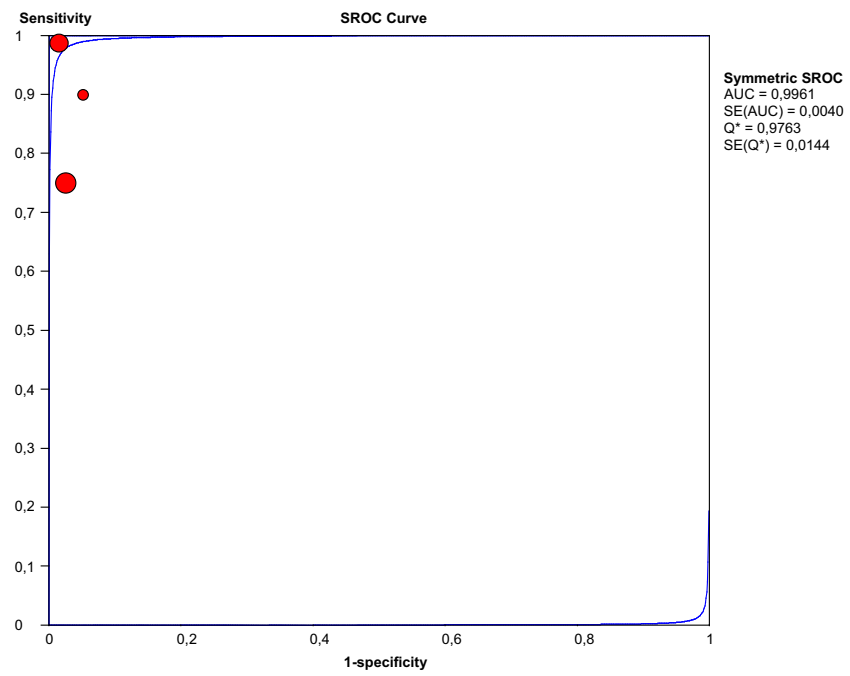

Fig. 4 Summary receiver operating characteristic curve of preoperative ultrasonography for detection of contralateral patent processus vaginalis in a complete cases and $\mathbf{b}$ incomplete cases. SROC summary receiver operating characteristic curve, AUC area under the curve 
Table 5 Diagnostic accuracy test results from incomplete cases included in the meta-analysis $(n=3)$. Incomplete cases: surgical exploration was only performed when the index test (preoperative ultrasonography) yielded positive test results. Clinical findings during follow-up (if reported) were used to calculate the amount of false negatives $(\mathrm{FN})$ and true negatives $(\mathrm{TN})$

\begin{tabular}{llrllllllllll}
\hline $\begin{array}{l}\text { Author, } \\
\text { year }\end{array}$ & $\begin{array}{l}\text { Patients } \\
(n)\end{array}$ & TP & FP & FN & TN & $\begin{array}{l}\text { Follow-up } \\
(\text { months })\end{array}$ & $\begin{array}{l}\text { Sensitivity } \\
(\%)\end{array}$ & $\begin{array}{l}\text { Specificity } \\
(\%)\end{array}$ & $\begin{array}{l}\text { PPV } \\
(\%)\end{array}$ & $\begin{array}{l}\text { NPV } \\
(\%)\end{array}$ & $\begin{array}{l}\text { US CPPV }^{\mathrm{a}} \\
(\%)\end{array}$ & $\begin{array}{l}\mathrm{Clinical} \mathrm{MCIH}^{\mathrm{b}} \\
(\%)\end{array}$ \\
\hline Erez, 1996 & 200 & 38 & 2 & 0 & 160 & $36-48$ & 100 & 98.8 & 95.0 & 100 & 20.0 & 19.0 \\
Uno, 1992 & 32 & 4 & 1 & 0 & 27 & $\geq 12$ & 100 & 96.4 & 80 & 100 & 15.6 & 12.5 \\
Zaidi, 2017 & 287 & 39 & 6 & 13 & 229 & $\leq 24$ & 75.0 & 97.4 & 86.7 & 94.6 & 15.7 & 18.1 \\
\hline
\end{tabular}

$T P$ true positive, $F P$ false positive, $F N$ false negative, $T N$ true negative, $P P V$ positive predictive value, $N P V$ negative predictive value, $U S$ ultrasound, $C P P V$ contralateral patent processus vaginalis, $M C I H$ metachronous contralateral inguinal hernia

${ }^{\text {a }}$ Amount (\%) of contralateral patent processus vaginalis as determined by ultrasonography

${ }^{\mathrm{b}}$ Amount (\%) of metachronous contralateral inguinal hernias that occurred during clinical follow-up

while others consider CPPV as potential hernia sac and include them in the category 'contralateral hernia' [5, 14, 23]. Thus, unlike others, Erez et al. used the hypoechoic width at the middle of the inguinal canal as the point of reference, instead of e.g. the maximum diameter or width of the inguinal canal at the internal ring [27]. A CPPV or contralateral hernia is further considered as the patency of the PV, as the presence of fluid, bowel loops or omentum in an open PV, or as the diameter of the internal ring exceeds a certain value (e.g. $\geq 4$ or $4.5 \mathrm{~mm}$ ). This shows the necessity of establishing more adequate homogeneous ultrasonographic criteria to accurately detect CPPV [28]. The results should be interpreted with caution because of high heterogeneity. Most studies included in our metaanalysis $(n=7)$ only explored the contralateral groin if preoperative ultrasonography yielded positive test results. This indicates a higher probability of the presence of CPPV during surgery, which could lead to an overestimation of the index test results. False negative rates of ultrasonography (FNR) for CPPV and FNR regarding the occurrence of MCIH might also be imprecise, since only four studies surgically explored all contralateral groins irrespective of the preoperative ultrasonographic test results [14-17] and only four studies have reported postoperative outcomes among patients who were not explored (Tables 1 and 2) [13, 18-20]. The use of different cut-off values to detect CPPV in the included studies might cause threshold effects to occur, also leading to heterogeneity of the results. Unfortunately, this meta-analysis did not provide enough data to explore the effects of differences in study characteristics on the estimates of the diagnostic test accuracy.

In conclusion, preoperative ultrasonography of the contralateral groin to detect CPPV seems promising, but may result in an overestimation of $\mathrm{MCIH}$ prevalence. More adequate, unequivocal ultrasonographic criteria are mandatory for proper diagnosis of CPPV and careful consideration should be given to what the appropriate reference standard is to establish accurate diagnosis of MCIH. Finally, risk factors need to be identified that can predict whether a CPPV actually develops into a clinically relevant $\mathrm{MCIH}$ or not.
Acknowledgements The authors would like to thank Faridi S. van EttenJamaludin for her contribution regarding the execution and updating of the search strategy and Alev Taner for the translation of a Turkish article.

Funding The authors state that this work has not received any funding.

\section{Compliance with ethical standards}

Guarantor The scientific guarantor of this publication is JPM Derikx, MD PhD.

Conflict of interest The authors of this manuscript declare no relationships with any companies whose products or services may be related to the subject matter of the article.

Statistics and biometry One of the authors, Professor JWR Twisk, has significant statistical expertise.

Informed consent Written informed consent was not required for this study because it comprises a systematic review of published literature.

Ethical approval Institutional review board approval was not required because it comprises a systematic review of published literature.

\section{Methodology}

- Diagnostic accuracy test systematic review and meta-analysis

Open Access This article is distributed under the terms of the Creative Commons Attribution 4.0 International License (http:// creativecommons.org/licenses/by/4.0/), which permits unrestricted use, distribution, and reproduction in any medium, provided you give appropriate credit to the original author(s) and the source, provide a link to the Creative Commons license, and indicate if changes were made.

\section{References}

1. Burgmeier C, Dreyhaupt J, Schier F (2014) Comparison of inguinal hernia and asymptomatic patent processus vaginalis in term and preterm infants. J Pediatr Surg 49:1416-1418

2. Chang SJ, Chen JYC, Hsu CK et al (2016) The incidence of inguinal hernia and associated risk factors of incarceration in pediatric 
inguinal hernia: a nation-wide longitudinal population-based study. Hernia 20:559-563

3. Steven M, Greene O, Nelson A, Brindley N (2010) Contralateral inguinal exploration in premature neonates: is it necessary? Pediatr Surg Int 26:703-706

4. Nataraja RM, Mahomed AA (2011) Systematic review for paediatric metachronous contralateral inguinal hernia: a decreasing concern. Pediatr Surg Int 27:953-961

5. Chen KC, Chu CC, Chou TY, Wu CJ (1998) Ultrasonography for inguinal hernias in boys. J Pediatr Surg 33:1784-1787

6. Toki A, Ogura K, Miyauchi A (1995) Ultrasonographic diagnosis of inguinal hernia in children. Pediatr Surg Int 10:541-543

7. Toki A, Watanabe Y, Sasaki K et al (2003) Ultrasonographic diagnosis for potential contralateral inguinal hernia in children. J Pediatr Surg 38:224-226

8. Andropoulos DB, Greene MF (2017) Anesthesia and developing brains - implications of the FDA warning. N Engl J Med 376:905907

9. Erdoğan D, Karaman İ, Aslan MK et al (2013) Analysis of 3,776 pediatric inguinal hernia and hydrocele cases in a tertiary center. J Pediatr Surg 48:1767-1772

10. Ein SH, Njere I, Ein A (2006) Six thousand three hundred sixty-one pediatric inguinal hernias: a 35-year review. J Pediatr Surg 41:980986

11. Hata S, Takahashi Y, Nakamura T et al (2004) Preoperative sonographic evaluation is a useful method of detecting contralateral patent processus vaginalis in pediatric patients with unilateral inguinal hernia. J Pediatr Surg 39:1396-1399

12. Kokorowski PJ, Wang HH, Routh JC et al (2013) Evaluation of the contralateral inguinal ring in clinically unilateral inguinal hernia: a systematic review and meta-analysis. Hernia. https://doi.org/10. 1007/s10029-013-1146-z

13. Kaneda H, Furuya T, Sugito K et al (2015) Preoperative ultrasonographic evaluation of the contralateral patent processus vaginalis at the level of the internal inguinal ring is useful for predicting contralateral inguinal hernias in children: a prospective analysis. Hernia 19:595-598

14. Chou TY, Chu CC, Diau GY et al (1996) Inguinal hernia in children: US versus exploratory surgery and intraoperative contralateral laparoscopy. Radiology 201:385-388

15. Kazez A, Serhatlioglu S, Akfirat M et al (1998) An alternative approach to contralateral exploration in infants with unilateral inguinal hernia: ultrasonography. Pediatr Cerrahi Derg 12:10-14
16. Lawrenz K, Hollman AS, Carachi R, Cacciaguerra S (1994) Ultrasound assessment of the contralateral groin in infants with unilateral inguinal hernia. Clin Radiol 49:546-548

17. Shehata SM, Ebeid AE, khalifa OM et al (2013) Prospective comparative assessment of ultrasonography and laparoscopy for contralateral patent processus vaginalis in inguinal hernia presented in the first year of life. Ann Pediatr Surg 9:6-10

18. Erez I, Rathaus V, Werner M et al (1996) Preoperative sonography of the inguinal canal prevents unnecessary contralateral exploration. Pediatr Surg Int 11:487-489

19. Uno T, Mochida Y, Wada H, Harada Y (1992) Ultrasonic exploration of contralateral side in pediatric patients with inguinal hernia. Surg Today 22:318-321

20. Zaidi SH, Rahman JU, Siddiqui TS et al (2017) Exploration of the contralateral groin in paediatric inguinal hernia or hydrocele based on ultrasound findings - is it justified? J Ayub Med Coll Abbottabad 29:26-29

21. Kazez AP (2001) The relation between patent processus vaginalis defined by ultrasonography and the clinical hernia: second step study. Pediatr Cerrahi Derg 15:107-110

22. Hasanuzzaman SM, Chowdhury LH, Sarker RN et al (2011) Ultrasonographic evaluation of contralateral exploration of patent processus vaginalis in unilateral inguinal hernia. Mymensingh Med J 20:192-196

23. Kervancioglu R, Bayram MM, Ertaskin I, Ozkur A (2000) Ultrasonographic evaluation of bilateral groins in children with unilateral inguinal hernia. Acta Radiol 41:653-657

24. Wang KS, Committee on Fetus and Newborn, American Academy of Pediatrics; Section on Surgery, American Academy of Pediatrics (2012) Assessment and management of inguinal hernia in infants. Pediatrics 130:768-773

25. Palmer LS (2013) Hernias and hydroceles. Pediatr Rev 34:457-464 quiz 464

26. Rowe MI, Copelson LW, Clatworthy HW (1969) The patent processus vaginalis and the inguinal hernia. J Pediatr Surg 4:102-107

27. Erez I, Rathause V, Vacian I et al (2002) Preoperative ultrasound and intraoperative findings of inguinal hernias in children: a prospective study of 642 children. J Pediatr Surg 37:865-868

28. Sameshima YT, Yamanari MG, Silva MA et al (2017) The challenging sonographic inguinal canal evaluation in neonates and children: an update of differential diagnoses. Pediatr Radiol 47:461-472 